\title{
mHealth through quantified-self: a user study
}

\author{
Chonlatee Khorakhun \\ University of St Andrews, UK \\ ck46@st-andrews.ac.uk
}

\author{
Saleem N. Bhatti \\ University of St Andrews, UK \\ saleem@st-andrews.ac.uk
}

\begin{abstract}
We describe a user study of a mHealth prototype system based on a wellbeing scenario, exploiting the quantifiedself approach to measurement and monitoring. We have used off-the-shelf equipment, with opensource, web-based, software, and exploiting the increasing popularity of smartphones and selfmeasurement devices in a user study. We emulate a mHealth scenario as a pre-clinical experiment, as a realistic alternative to a clinical scenario, with reduced risk to sensitive patient medical data. We discuss the efficacy of this approach for future mHealth systems for remote monitoring. Our system used the popular Fitbit device for monitoring personal wellbeing data, the Diaspora online social media platform (OSMP), and a simple Android/iOS remote notification application. We implemented remote monitoring, asynchronous user interaction, multiple actors, and usercontrolled security and privacy mechanisms. We propose that the use of a quantified-self approach to $\mathrm{mHealth}$ is particularly valuable to undertake research and systems development.
\end{abstract}

\section{INTRODUCTION}

The use of an online social media platform (OSMP) to enable pervasive and seamless health monitoring has already been proposed in our previous works [1]-[4]. By exploiting existing, publicly accessible infrastructure, coupled with the use of an OSMP, a healthcare provider could benefit from reduced costs of implementation and deployment, compared to a fully custom-made solution. However, experimentation within a clinical scenario can remain costly, time-consuming, and have risks due to the use of sensitive medical data. While clinical trials are essential before real deployment, we propose that early stages of experimentation can benefit from preclinical user studies.

Our contribution in this work is two-fold. First, we describe a prototype experiment for using an opensource OSMP Diaspora - for mHealth monitoring and alert. Second, we demonstrate how wellbeing data generated from a personal device - Fitbit - can be used to replace sensitive medical data, to provide useful feedback on systems development in a pre-clinical experiment.

After discussing related work in Section II, we describe in Section III a remote monitoring application (RMA) using a Fitbit device and the Diaspora OSMP. The description of the user study and the RMA model are presented in Section IV and V respectively. A discussion of our findings from the user study is given in Section VI. Finally, we conclude with a summary in Section VII.

\section{RELATED WORK}

\section{A. Meeting challenges in developing mHealth systems}

Development of mHealth systems has been dogged by a combination of various factors: high costs; low uptake after trials; use of costly, customised devices; and poor usability of systems [5]-[7]. The use of sensitive, personal medical data also presents a key challenge in conducting experiment for healthcare research, as does the involvement of professional medical staff and real patients. Many ethical, medical and pastoral concerns that are complex need to be managed. The trials in [8]-[11] are good examples of mHealth monitoring experiments which use sensitive health data, in high-risk environments, and require clinicians to be involved.

However, for a pre-clinical setting, to investigate technology and systems interaction, such complexity from clinical involvement could be removed without losing context and relevance to the eventual clinical application by the use of a wellbeing scenario [12].

\section{B. The quantified self}

The availability of technologies in self-monitoring result in a variety of applications and devices for health and fitness monitoring, e.g. FitBit [13], Jawbone UP [14], Nike+ [15], Samsung Gear [16], Apple Watch [17], etc.

In our study, a Fitbit device was used for collecting measurements. This is one of the most popular [18] and accurate [19] activity trackers. It provides accessible public APIs and has been used in many research studies, e.g. [20]-[23], including in a clinical setting for remote health monitoring [24].

\section{The use of OSNs in healthcare}

Due to the sensitivity of health data and various legal regulations, the use of online social network (OSN) systems for building private healthcare systems might appear to be infeasible. So, previous use of OSNs has been limited to specific aspects of healthcare, e.g. Patientslikeme [25] is a platform for patients only to share experiences; Doximity [26] and Sermo [27] provide similar facilities for doctors only; and HelloHealth [28] is a private platform for maintaining Electronic Health Record (EHR) data.

The use of an OSN can enable efficient and timely collaboration and communication for patients and healthcare professionals in a healthcare network. We have shown that it is feasible to achieve security and privacy of private health data as well as considerable functionality for mHealth monitoring by the use of a completely opensource OSMP [3]. 


\section{Privacy and data sharing}

The quantified-self and self-monitoring raise concerns and issues for privacy and data sharing, e.g. with whom and to which extent people are willing to share their private health data. Most of the previous studies on privacy and data sharing in healthcare, e.g. [29], [30], have been based on interviews and focused on sharing of health data in general. The first study in this area which collects data with real user interactions is by Prasad et al [22]. However, such studies are resource intensive, difficult to execute and reproduce, and can take a considerable amount of time.

We were interested in studying the sharing behaviours of people in a controlled and secure environment of a carer network [4]. We have considered privacy concerns and sharing of sensitive data with trusted parties in a healthcare regime that is closed with respect to the actors involved. Our approach is designed with the goals of simplicity and reproducibility, while enabling the investigation of significant challenges, such as data sharing, security and privacy.

\section{WELlbeING AS A PROXY FOR MHEALTH}

Our experiment was designed using a wellbeing scenario as a proxy of mHealth monitoring, with equivalent roles for the carer network.

\section{A. Carer network}

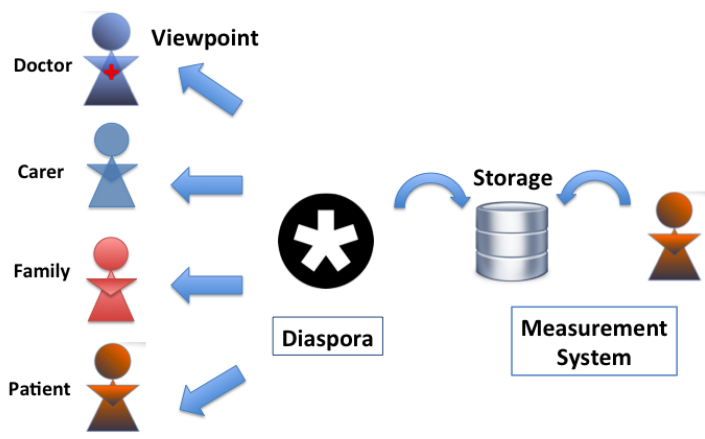

Fig. 1: A design of a wellbeing experiment as a proxy for mHealth monitoring using an online social network (OSN). Fitbit is used as a measurement system. An opensource online social media platform (OSMP) - Diaspora - provides access to the stored Fitbit data for different actor viewpoints. The conventional actors in a carer network (patient, family, carer and doctor) are mapped to parallel roles (client, fitness buddy, fitness coach and personal trainer) in our wellbeing scenario.

In Figure 1, our remote monitoring application (RMA) provides a portal to access the collected bio-data and provide the appropriate viewpoints for actors in a carer network [1], [2]. Based on the relationships in an existing healthcare regime that is common worldwide [31], the carer network includes both informal and formal caregivers, works in harmony with, and provides support for, existing relationships and interactions between patients and healthcare professionals:

1) the doctor or consultant in charge of the management of the healthcare regime;
2) the professional carer who is a local contact for the patient and implements the clinical care (e.g. General Practitioner in the UK);

3) a family member or friend who is concerned about the patient and acts as an informal caregiver (e.g. a neighbour for an elderly patient);

4) and the patient.

\section{B. Fitness network}

The welbeing network is set up as a parallel network to the carer network [12]. The equivalent roles are:

1) A client. This role is equivalent to the patient in the carer network. The client uses Fitbit and Diaspora to improve their health and fitness.

2) Personal trainer. This role is equivalent to the doctor in the carer network. The trainer is a fitness professional, e.g. staff from a gym, and has responsibility for designing exercise plans and setting goals subject to the clients' needs and abilities.

3) Fitness coach. This is equivalent to role of the carer, who might be a local healthcare professional at a clinic, or local physician.

4) Fitness buddy. This role is equivalent to the informal caregiver like a friend or family member who helps the client to work toward their goals by monitoring the client's performance, motivating and/or working together.

In reality, the professional roles, i.e. trainer and coach functions as well as the doctor and carer functions, might be fulfilled by the same person. This would depend on the nature of the medical situation.

\section{Analogy in data sharing}

There is a clear equivalence between our wellbeing monitoring scenario and mHealth monitoring, in terms of measurement and sharing of data. Similar to patients using wearable sensors to collect their bio-data, our experiment used Fitbit devices to collect steps and sleep patterns. Like patients, our clients had control over the collection of their data and made decisions on how to share their data.

Data sharing behaviour in both scenarios are subject to purpose-oriented sharing. Accordingly, clients shared their activity data with the group of trusted people in the wellbeing network for the purpose of improving their fitness levels, while patients would share their health data in a carer network for medical treatment and/or diagnosis. It is noted that sharing in both scenarios is based on scenario-specific trust relationships, and unlike sharing financial data or private information to third parties in general online social networks, e.g. Facebook.

\section{USER STUDY}

Previously [3], we have investigated the use of an opensource OSMP - Diaspora - a platform to enable personal health monitoring while enabling flexible application development, allowing fine-grained control of security \& privacy. 
The mapping from carer network to the wellbeing network (trainer, coach, buddy and client) can be realised.

The Fitbit Charge HR activity tracker (Figure 2) was used as a measurement device. It measured the steps walked, sleep data and heart rate for a wearer, uploading data to the Fitbit server via a smartphone. Our RMA (built on Diaspora) used the Fitbit API to access the data from the Fitbit server and stored it locally. Each actor had a different viewpoint of monitored data, and different levels of control of the RMA, depending on their roles in the wellbeing/carer network. Both Google Clound Messaging (GCM) for Android and Apple Push Notifications (APNs) for iOS were implemented via the RMA.

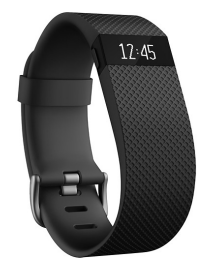

Fig. 2: Fitbit Charge HR wristband device used for measuring steps walked, sleep data and heart rate.

\section{A. Study design}

Table I shows the week-by-week activities as part of the study design. There were two weeks of preparation: one week for recruitment and one week for training. The main study was conducted in over 9 weeks. During the period of the study, a set of user surveys were completed by all participants: a survey on their background (week 01); surveys on functionality and privacy (weeks 04, 06, 09 and 12), and a survey on feedback after users had returned their Fitbit devices.

Participants were paired so each could play a buddy and client role in their pairs. At weeks 05, 08 and 11, the pairs were re-arranged with a randomly selected participant and worked as a new pair for a period of one week. The buddy swap was to investigate behaviour in sharing data with other participants.

Collaboration vs competition. According to [32], if the context of sharing fits the goals, external competition together with the internal collaboration is most effective for participants. Therefore, in our experiment, the client and buddy in each pair worked together as a team, each acting as both buddy and client to each other, forming two logical fitness/carer networks. They both can monitor each other's Fitbit data, and work in a collaborative manner to compete with other client-buddy pairs.

Sharing of sensitive but useful data. Within the context of the trusted environment of the wellbeing/carer network, the interview in [33] shows that people are willing to share their sensitive health data if the data is seen as useful in helping to provide care. So, in our experiment, users have control over sharing of their sleep and heart rate data which is considered as useful data for wellbeing but not strictly necessary for goals achievement, even though it could be relevant data as indicators of health status if goals are not being met [34]. All default sharing is opt-out, i.e. the sleep data is shared with both buddy and professionals and the heart rate data is shared with professionals at the beginning of the study. Over the course of the study, clients can decide to turn off their sharing either for buddy or professional, and the change in settings was recorded.

Sharing behaviour. At the end of the study, clients can state the reasons behind their sharing behaviours, e.g. if there is a difference in trust between professional and personal sharing, or if there is an influence from a privacy setting of a client's buddy. In order to check the stated behaviour against actions taken by the user, the steps and sleep data are also recorded and used for analyses of sharing behaviour. For example, clients might turn off sharing just because they do not walk well or sleep well during a period and are reluctant to let their buddy know they are failing to meet goals.

\section{B. Surveys}

Four types of survey were used (Table I):

1) Background survey.: This was conducted prior to the study to assess participants' characteristics prior to taking part in the study, e.g. physical activity and interest in technology. This also included the experience of participants with OSNs and quantified-self systems, as well as daily physical activities (how much they normally walk per day). Also, the survey recorded self-assessment of participants' motivation toward walking and being active.

2) Feedback survey.: This was conducted at the end of the study to assess the overall improvement participants experienced during the study, as well as the participants' acceptance in using the RMA in our wellbeing scenario. Our aim was to assess whether the use of the carer/wellbeing network helped to improve their wellbeing.

3) Survey on functionality.: This was conducted four times over the course of the study to investigate the usability of the platform for monitoring and issuing alerts, as well as the usefulness of the wellbeing network. We assessed how monitoring and alerts along with having the professionals and buddy access participants' bio-data helped participants to improve their wellbeing and drive them to work toward goals.

4) Survey on privacy.: This was conducted four times over the course of the study to investigate participants' data-sharing behaviour and preferences with respect to professionals and buddies. We also wanted to assess if participants felt they would respond differently in a real clinical scenario compared to our wellbeing scenario.

\section{User assessment metrics}

For evaluation of the user study, we defined index values for measuring participants' assessments. This applied to the range of responses from the survey questions that used a Likert scale: not at all (1), not much (2), undecided (3), somewhat (4) and very much (5). We defined the User Positive Index (UPI), User Negative Index (UNI) and User Undecided Index (UUI):

$$
\begin{aligned}
U P I & =[n(5)+n(4)] / n(\text { total }) \\
U N I & =[n(1)+n(2)] / n(\text { total }) \\
U U I & =n(3) / n(\text { total })
\end{aligned}
$$




\begin{tabular}{|l|c|c|c|c|c|c|c|c|c|c|c|c|c|}
\hline Week & 01 & 02 & 03 & 04 & 05 & 06 & 07 & 08 & 09 & 10 & 11 & 12 & 13 \\
\hline Recruitment & $\diamond$ & & & & & & & & & & & & \\
\hline Training & & $\diamond$ & & & & & & & & & & & \\
\hline User study & & & $\diamond$ & $\diamond$ & $\diamond$ & $\diamond$ & $\diamond$ & $\diamond$ & $\diamond$ & $\diamond$ & $\diamond$ & & \\
\hline Buddy swaps & & $\mathrm{O}$ & $\mathrm{O}$ & $\mathrm{O}$ & $\mathrm{S}$ & $\mathrm{O}$ & $\mathrm{O}$ & $\mathrm{S}$ & $\mathrm{O}$ & $\mathrm{O}$ & $\mathrm{S}$ & & \\
\hline Surveys & & & $\mathrm{B}$ & 1 & & 2 & & & 3 & & & 4 & $\mathrm{~F}$ \\
\hline
\end{tabular}

TABLE I: The study lasted for 9 weeks, with 1 week of recruitment and 1 week of training. Each participant was paired with another participant - a buddy. Buddy swaps: $\mathrm{O}$ is original buddy; $\mathrm{S}$ is a new, randomly-chosen buddy. Surveys: $\mathrm{B}$ is a background survey; F is a feedback survey; number $N$ indicates $N$ th survey of functionality and privacy.

where $n(i)$ is the number of participant responses to value $i$ and $n($ total $)$ is the total number of participants. The closer the value of $U P I$ (positive), $U N I$ (negative) or $U U I$ (undecided) is to 1 , the stronger is that response.

\section{RMA FOR MHEALTH PROXY}

The open-source OSMP Diaspora was modified with RMA functionality, to demonstrate the following: remote monitoring; asynchronous alerts; multiple actors in a healthcare regime; and appropriate security and privacy mechanisms. RMA was web-based [35] secured using HTTPS.

Two primitive RMA functionalities were implemented: remote monitoring and asynchronous alerts. As shown in Figure 3a, the alert is implemented as a Diaspora message, which is triggered when the predefined condition is met, e.g. goals are achieved, configurations are changed, or Fitbit data is out of date.

The level of access to the collected Fitbit data as well as the level of control of the application was restricted by the role of the actors in the fitness/carer network. A patient (client) had access to their own Fitbit data, their buddy's data and their sharing preferences, i.e. to select which information to share with whom in the fitness network.

Figures $3 \mathrm{~b}$ and $3 \mathrm{c}$ show example RMA information for patient (client) and doctor (trainer) viewpoints, respectively. The presentations consist of different visualisations, e.g. tables, meters and charts. Note that the chart and meter graphics were from Google Charts to demonstrate the use of a mashup for fast application development. To avoid privacy concerns with bio-data in a real $\mathrm{mHealth}$ application, such visualisations would be provided by the healthcare provider or a trusted party and not by Google Charts.

In these examples, the client (patient) as well as the buddy (family) can access only the monitored data for that day, whereas the doctor (trainer) and the carer (coach) have more detailed access to all historical Fitbit data. Also, the level of control and management which the doctor (trainer) has over the application ranges from being able to change goals, to control of monitoring functions.

All views are controlled by policy, so are configurable as required: we present a simple example to show the concept. Each actor has access only to the part of the data they require subject to their roles in the carer (fitness) network. Our Diaspora application is used as a portal to authorise each actor and provide an appropriate viewpoint and visualisations.

Since our application is web-based access, an additional mobile application was implemented in order to receive mobile
TABLE II: Summary of participants' background.

\begin{tabular}{ll}
\hline Total number of participants & 17 \\
\hline Gender & \\
Male & 11 \\
Female & 6 \\
\hline Occupation & \\
PhD student & 13 \\
Master student & 1 \\
University staff & 2 \\
Non-university member & 1 \\
\hline
\end{tabular}

push notifications in addition to in-platform Diaspora notifications. Both Google Cloud Messaging (GCM) for Android and Apple Push Notification service (APNs) were implemented and used for our user study.

\section{DISCUSSION}

As shown in Table II, 17 participants were recruited. All participants were motivated to take part in the study and engaged well. They were all volunteers: enthusiastic to try new technology to help improve their fitness and wellbeing. All participants were experienced in using online social media, and all were active users, i.e. accessing OSNs many times a day. Some of the participants had experience with having a coach or trainer, while most of them had experience with using quantified-self applications, e.g. Nike+, Fitbit, etc.

\section{A. Change in participant motivation}

Figure 4 shows a comparison of participants' selfassessment for motivation to walk before and after the study. Form the UPI and UNI values, we see that participants were more interested in their own activities after the study, i.e. an increase in UPI with decrease in UNI after the study. In addition, Figure 4 shows a slight increase in motivation to walk from having professionals to monitor their activities, with increase in UPI. This means most participants liked that they had professionals monitoring, and their opinions are consistent, i.e. they are equally motivated by having professionals before and after the study. However, there is a decrease in importance and usefulness of having a workout buddy, with a decrease in UPI and an increase of UNI.

We observed that, initially, people need a buddy to motivate them to engage with the study, but, once they are engaged, the buddy's importance decreases. We infer that in a mHealth scenario, patients may need encouragement from a family member or friend to engage with a mHealth care regime. 


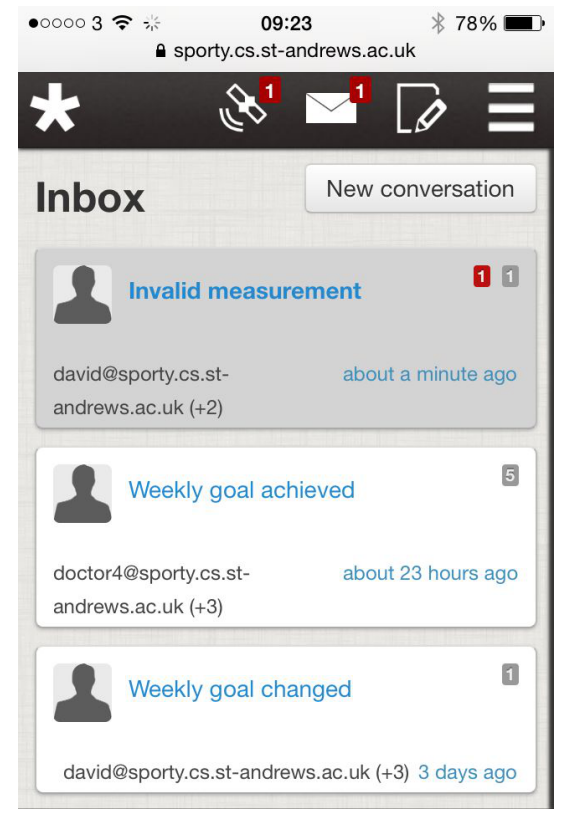

(a) Notification alerts sent in the form of Diaspora messages.

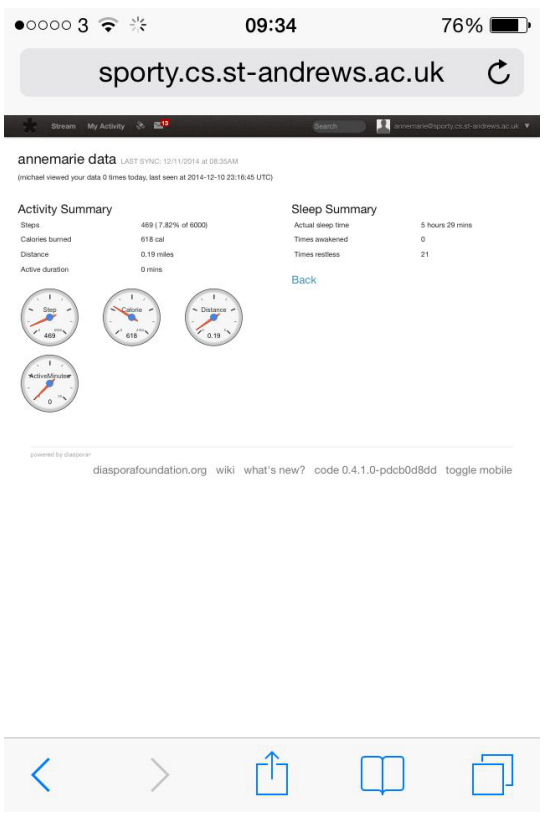

(b) Patient Fitbit data viewpoint. No detailed access to Fitbit data.

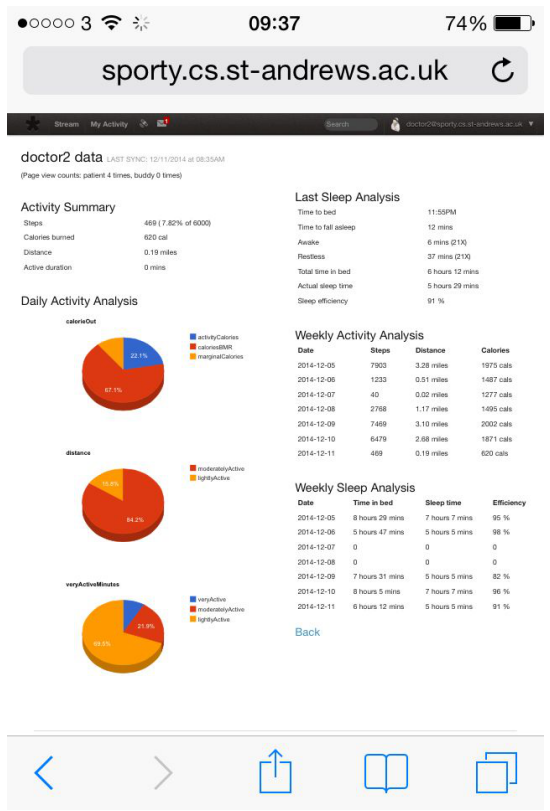

(c) Doctor Fitbit data viewpoint. Full detailed access to Fitbit data.

Fig. 3: Example Diaspora RMA screenshots. We show a view from a mobile device, but as we have a web-based interface, non-mobile devices can also access the data in a consistent manner. All views are configurable subject to appropriate policy: we have presented simple examples here to show the concept.

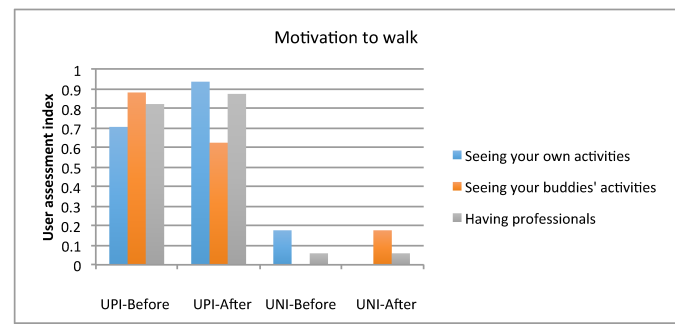

Fig. 4: Comparison of participants' self-assessments on motivation to walk, before and after the study.

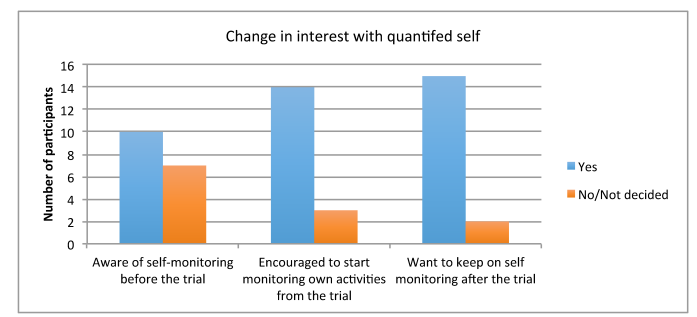

Fig. 5: Users gain interest in quantified-self as a result of participation. At the end of the study, 15 out of 17 participants would like to continue self-monitoring.

\section{B. Increased interest in quantified-self}

Figure 5 shows that participants gained interest in selfmonitoring. Before the study, 10 out of 17 participants were already aware of self-monitoring. By involvement in the study, 14 out of 17 participants said there were encouraged to start monitoring their own activities. After the study, when the Fitbit devices were returned, 15 out of 17 participants said they would continue self-monitoring ( 8 of them bought their own Fitbit device or a similar self-monitoring device, 2 of them already had similar devices, and 5 of them would continue when they could purchase a device at an affordable price).

We infer that allowing patients to self-monitor (see their own data) could encourage them to engage and stay engaged with mHealth systems.

\section{Participant acceptance of the RMA}

Figure 6 shows participants' assessment of the monitoring functionality in our Diaspora platform at the end of the study. The responses are highly positive. Figure 7 shows participants' assessment of three platform functions, in which most participants (13 out of 17) also agreed that the monitoring functionality in the Diaspora platform was useful, while none of the participants disagreed with that. For notifications, most participants (12 out of 17) agreed that to receive Diaspora notifications was useful. Most participants (14 out of 17) agreed that the wellbeing network was useful for improving their fitness/wellbeing.

We infer that the success of mHealth systems could be improved by allowing self monitoring, notifications for patients, and linkage between patients and carers.

\section{Utility of the OSN for RMA}

We traced participants' responses in how the RMA platform is important in improving the following aspects of remote 


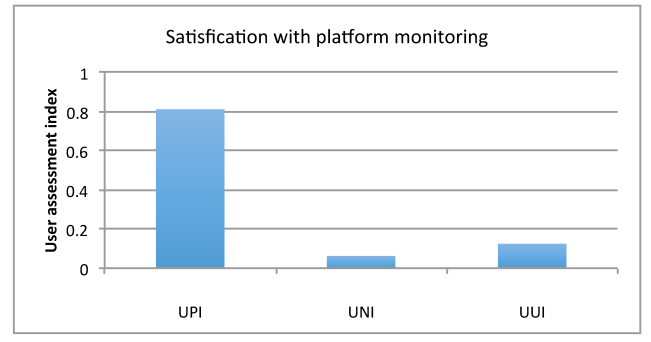

Fig. 6: Participants' assessment of the RMA for monitoring functionality at the end of the study, i.e. to what extent the RMA fulfilled the monitoring of daily steps/walking.

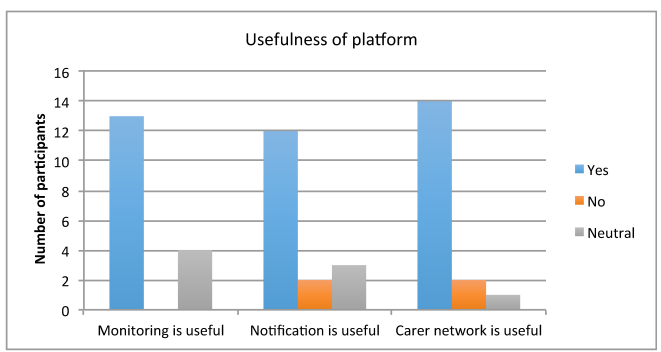

Fig. 7: Participants' assessment of the usefulness of the RMA functionality at the end of the study. Yes = platform functionality was useful. No = platform functionality was not useful. Neutral $=$ undecided.

monitoring: providing closer communication with professionals and buddy, and allowing communication of bio-data. As shown in Figure 8, most participants see the importance of both of these aspects (more positive ratings than negative ones). Note that Figure $8 \mathrm{~b}$ shows a decrease in the usefulness of communication of bio-data experienced by participants over the survey. Together with Figure 4, this result shows that people like being engaged with a professional, but lose interest in their buddies, i.e. they are more interested in self-monitoring and having engagement with professionals.

We infer that patients will be motivated to stay engaged with a mHealth regime through communication and linkages enabled by the carer network. However, they will prefer communication with the care professionals.

\section{E. Sharing preference in quantified-self}

We assessed participants' opinions on the utility of having control over sharing of their data. As shown in Figure 9, most participants (12 out of 17) agreed that the interface was useful for them to share information, either with their buddies or professionals. Participants were satisfied with the default setting provided in our scenario and therefore made no change to their settings. Nevertheless, they did realise the importance of having a control for sharing of their data.

We assessed participants' opinions on sharing preferences. Figure 10 shows that most participants (11 out of 17) preferred to share sensitive data with professionals, and none of the participants wanted to share more with their buddies.

The sharing behaviour in our scenario is similar to the medical scenario in general, i.e. patients have trust with, and

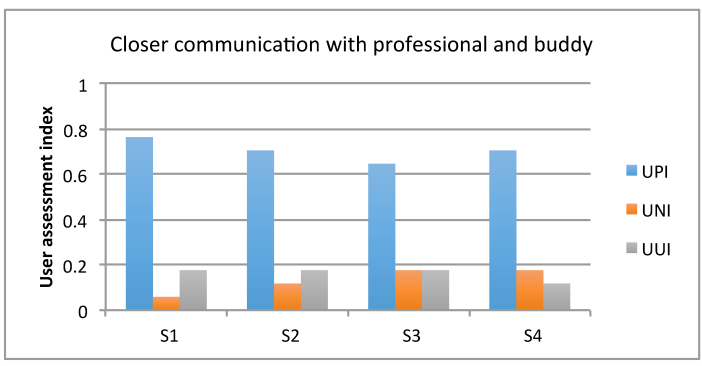

(a) Allow closer communication with professional and buddy

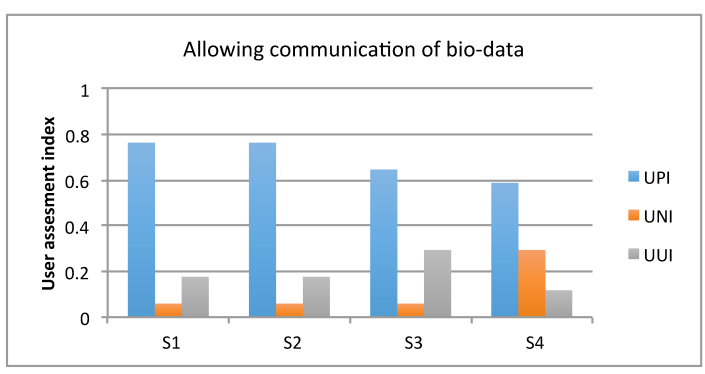

(b) Allow communication of bio-data

Fig. 8: Participants' assessment of the usefulness of the RMA based on social interaction. $\mathrm{S} n$ is survey $N$ (see Table I).

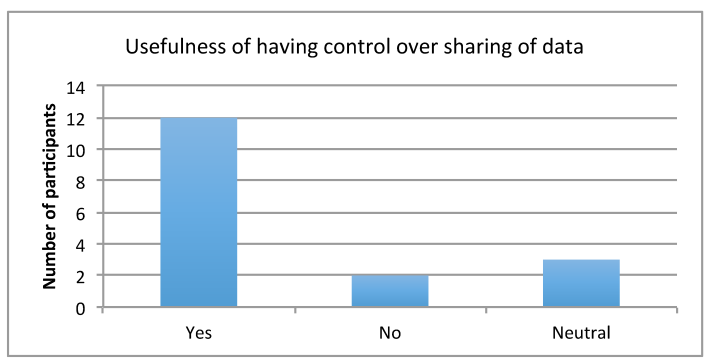

Fig. 9: Participants' opinions on usefulness of having control over sharing of their data (heart rate and sleep). Yes $=$ it is useful to have control over sharing of data. No $=$ it is not useful to have control over sharing of data. Neutral $=$ undecided.

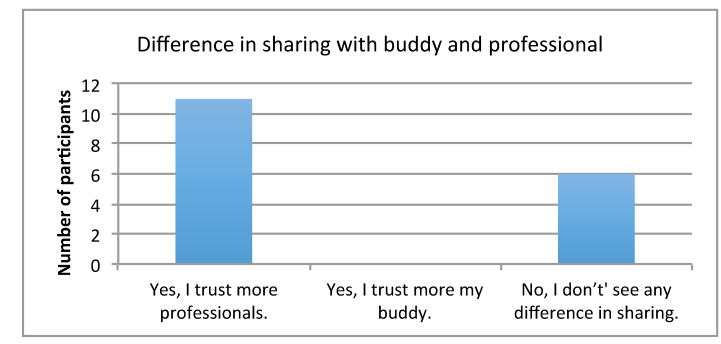

Fig. 10: Participants' opinions on difference between sharing their sensitive data with buddies and professionals.

will share their data with, health professionals, while having controls over what information they share with professionals.

However, this will not be the same for all medical scenarios: it is difficult to generalise the medical situation. For example, comparing two diseases like asthma and AIDS, people would have different sensitivity in sharing. People having asthma 
might be more willing to share their information, while people having AIDS might be less willing to share their information.

\section{CONCLUSION}

We have designed and built a mHealth remote monitoring application (RMA) using an opensource online social media platform (OSMP) and a consumer self-monitoring device. The opensource RMA functionality included asynchronous alerts as well as monitoring of quantified-self data. We used the quantified-self approach to create a proxy for a mHealth scenario, and have experimented with a pre-clinical application without involving sensitive medical data from real patients, but without loss of context.

Our user study highlights issues to be considered in specific situations. However, we find a good correspondence between the wellbeing scenario and a mHealth scenario, by our use of a carer network - a socially-related group of actors in a typical healthcare regime.

We believe that the use of wellbeing monitoring as a proxy for mHealth monitoring would more easily facilitate user studies and trials in the early research stages of mHealth applications, to make faster progress of advancing future mHealth systems. From our results, we also propose that mHealth systems could be more successfully developed and deployed if users are more engaged by having visibility of their own data and being given control of who sees that data.

\section{ACKNOWLEDGEMENTS}

This work was partly supported by the IU-ATC project, funded by grant EP/J016756/1 from the Engineering and Physical Sciences Research Council (EPSRC). Chonlatee Khorakhun is funded by the Scottish Informatics and Computer Science Alliance (SICSA).

\section{REFERENCES}

[1] C. Khorakhun and S. N. Bhatti, "Remote Health Monitoring Using Online Social Media Systems," in Proc. 6th IFIP/IEEE Wireless and Mobile Networking Conference (WMNC2013), Apr 2013.

[2] _ , "Alerts for Remote Health Monitoring Using Online Social Media Platforms," in IEEE HealthCom 2013, Oct 2013

[3] _ , "Using Online Social Media Platforms for Ubiquitous, Personal Health Monitoring," in IEEE HealthCom 2014, Oct 2014.

[4] — , "Remote health monitoring using online social media," EAI Endorsed Trans. on Ubiquitous Environments, vol. 14, no. 03, Nov 2014

[5] P. Yu, M. X. Wu, H. Yu, and G. Q. Xiao, "The challenges for the adoption of m-health," in SOLI 2006 - IEEE Intl. Conf. Service Operations, Logistics, and Informatics. IEEE, 2006, pp. 181-186.

[6] P. Mechael, H. Batavia, N. Kaonga, S. Searle, A. Kwan, A. Goldberger, L. Fu, and J. Ossman, Barriers and gaps affecting mHealth in low and middle income countries: Policy white paper. Columbia University. Earth Institute. Center for Global Health and Economic Development (CGHED): with mHealth Alliance, 2010.

[7] W. Chigona, M. Nyemba, and A. Metfula, "A review on mhealth research in developing countries," Jrnl. of Community Informatics, vol. 9, no. 2, 2012.

[8] U. Anliker, J. A. Ward, P. Lukowicz, G. Troster, F. Dolveck, M. Baer, F. Keita, E. B. Schenker, F. Catarsi, L. Coluccini et al., "AMON: a wearable multiparameter medical monitoring and alert system," IEEE Trans. Inf. Tech. in Biomedicine, vol. 8, no. 4, pp. 415-427, 2004.
[9] M. Blount, V. M. Batra, A. N. Capella, M. R. Ebling, W. F. Jerome, S. M. Martin, M. Nidd, M. R. Niemi, and S. P. Wright, "Remote healthcare monitoring using Personal Care Connect," IBM Systems Journal, vol. 46, no. 1, pp. 95-113, 2007.

[10] A. Wood, G. Virone, T. Doan, Q. Cao, L. Selavo, Y. Wu, L. Fang, Z. He, S. Lin, and J. Stankovic, "ALARM-NET: Wireless sensor networks for assisted-living and residential monitoring," University of Virginia Computer Science Department Technical Report, vol. 2, 2006.

[11] G. R. Hayes, K. G. Cheng, S. H. Hirano, K. P. Tang, M. S. Nagel, and D. E. Baker, "Estrellita: A mobile capture and access tool for the support of preterm infants and their caregivers," ACM Trans. on Computer-Human Interaction (TOCHI), vol. 21, no. 3, p. 19, 2014.

[12] C. Khorakhun and S. N. Bhatti, "Wellbeing as a proxy for a mHealth study," in IEEE Wkshp. The Role of Quantified Self for Personal Healthcare (QSPH), Nov 2014.

[13] "FitBit," http://www.fitbit.com, (Dec 2014).

[14] "Jawbone Up," https://jawbone.com/up, (Dec 2014).

[15] “Nike+," https://secure-nikeplus.nike.com/plus/, (Dec 2014).

[16] "Samsung gear," http://goo.gl/qCTK3Q, (Dec 2014).

[17] “Apple watch," https://www.apple.com/watch/, (Dec 2014).

[18] Dara Kerr, "Fitbit rules 50 percent of the world's wearable market," http://www.cnet.com/news/fitbit-rules-50-percent-of-theworlds-wearable-market/, (May 2014).

[19] F. Guo, Y. Li, M. S. Kankanhalli, and M. S. Brown, "An evaluation of wearable activity monitoring devices," in Proc. 1st ACM Intl. Workshop on Personal data meets distributed multimedia. ACM, 2013, pp. 31-34.

[20] G. Walsh and J. Golbeck, "Stepcity: a preliminary investigation of a personal informatics-based social game on behavior change," in CHI'14 Ext. Abstracts on Human Factors in Comp. Sys. ACM, 2014, pp. 2371-2376

[21] P. Pendse and J. Greene, "A wellness android application with social networking capabilities," in Proc. 51st ACM Southeast Conference. ACM, 2013, p. 24.

[22] A. Prasad, J. Sorber, T. Stablein, D. Anthony, and D. Kotz, "Understanding sharing preferences and behavior for mHealth devices," in Proc. ACM Wrkshp. Privacy in the electronic society, 2012, pp. 117-128.

[23] K. Tollmar, F. Bentley, and C. Viedma, "Mobile Health Mashups," in Proc. PervasiveHealth 2012, May 2012.

[24] Jonah Comstock, "Samsung reveals Simband and Sami health platform," http://mobihealthnews.com/25126/mayo-fitbit-data-predictssurgical-recovery-time/, (Aug 2013).

[25] "patientslikeme," http://www.patientslikeme.com, (Sep 2014).

[26] "Doximity," https://www.doximity.com, (Sep 2014).

[27] "Sermo," http://www.sermo.com, (Sep 2014).

[28] "hellohealth," http://hellohealth.com, (Sep 2014).

[29] M. W. Newman, D. Lauterbach, S. A. Munson, P. Resnick, and M. E. Morris, "It's not that I don't have problems, I'm just not putting them on Facebook: challenges and opportunities in using online social networks for health," in Proc. ACM CSCW 2011. ACM, 2011, pp. 341-350.

[30] D. A. Epstein, A. Borning, and J. Fogarty, "Fine-grained sharing of sensed physical activity: a value sensitive approach," in Proc. 2013 ACM Intl. Conf. Pervasive and Ubiquitous Comp. ACM, 2013, pp. 489-498.

[31] N. Carpentier and F. Ducharme, "Care-giver network transformations: the need for an integrated perspective," Ageing and Society, vol. 23, no. 04, pp. 507-525, 2003.

[32] T. Y. J. N. K. T. Yuuki Nishiyama, Tadashi Okoshi and H. Tokuda, "Towards Health Exercise Behavior Change for Teams Using Lifelogging," in IEEE HealthCom 2014, Oct 2014.

[33] K. Wild, L. Boise, J. Lundell, and A. Foucek, "Unobtrusive in-home monitoring of cognitive and physical health: Reactions and perceptions of older adults," Journal of Applied Gerontology, vol. 27, no. 2, pp. 181-200, 2008.

[34] G. G. Alvarez and N. T. Ayas, "The impact of daily sleep duration on health: a review of the literature," Progress in cardiovascular nursing, vol. 19, no. 2, pp. 56-59, 2004.

[35] B. Frain, Responsive Web Design with HTML5 and CSS3. Packt Publishing, Apr 2012. 